\title{
Evaluation of MODIS and Sentinel-3 SLSTR Thermal Emissive Bands calibration consistency using Dome C
}

\author{
Ashish Shrestha $^{\mathrm{a}}$, Amit Angal ${ }^{\mathrm{a}}$, and Xiaoxiong Xiong ${ }^{\mathrm{b}}$ \\ ascience Systems and Applications, Inc., Lanham, MD 20706 USA \\ bNASA Goddard Space Flight Center, Greenbelt, MD, 20771 USA
}

\begin{abstract}
Over the years, data from different satellites has provided invaluable information about Earth's atmosphere, land and oceans. The thermal emissive bands (TEB) on the Moderate Resolution Imaging Spectroradiometer (MODIS) are comprised of 16 spectral bands with wavelengths ranging from 3.7 to $14.4 \mu \mathrm{m}$. MODIS TEB are calibrated on orbit on a scan-by-scan basis using an on-board blackbody (BB). Sentinel-3 Sea and Land Surface Temperature Radiometer (SLSTR), launched on 16 February 2016, has 11 spectral bands with wavelengths from 0.55 to $12 \mu \mathrm{m}$. In this study, we compare the observed brightness temperature from MODIS bands 31 and 32 and SLSTR bands S8 and S9 over Dome C using a $20 \times 20 \mathrm{~km}$ region of interest (ROI) centered at $\left(75.102^{\circ}\right.$ $\left.\mathrm{S}, 123.395^{\circ} \mathrm{E}\right)$. A total of 2989 scenes for Terra, 2963 for Aqua and 1961 for SLSTR from November, 2016 to January 2018 are analyzed. The relative bias between MODIS and SLSTR is evaluated using the near-surface temperature measurements from an Automatic Weather Station (AWS).
\end{abstract}

Keywords: MODIS, SLSTR, thermal emissive bands, calibration, Dome C

\section{INTRODUCTION}

Over the years, data from different airborne and spaceborne sensors has provided invaluable information for scientists to study the Earth's atmosphere, land and oceans. It is important to have data from these different sensors on a same radiometric scale, hence instruments calibration is very important. The Moderate Resolution Imaging Spectroradiometer (MODIS) is one of the key instrument on-board NASA's Terra and Aqua satellites. ${ }^{1}$ Terra and Aqua MODIS have successfully completed 18 and 16 years of on-orbit operation respectively. MODIS is a cross-track, whisk-broom, scanning imaging radiometer that uses a double-sided scan mirror to obtain nearly continuous images of the Earth with a scan angle range of $\pm 55^{\circ}$ at an altitude of $705 \mathrm{~km} .{ }^{2}$ MODIS has 36 spectral bands with wavelengths ranging from 0.4 to $14.4 \mu \mathrm{m}$. Among those 36 bands, bands $1-19$ and 26 are the reflective solar bands $(\mathrm{RSB})(<2.2 \mu \mathrm{m})$ and bands $20-25$ and $27-36$ are the thermal emissive bands (TEB) $(>3.6 \mu \mathrm{m})$. The spatial resolutions for the RSB are $250 \mathrm{~m}$ for bands 1 and 2, $500 \mathrm{~m}$ for bands $3-7$ and $1000 \mathrm{~m}$ for bands $8-19$ and band 26, while all TEB have $1000 \mathrm{~m}$ resolution at nadir. The MODIS bands are located on one of four focal plane assemblies (FPA); the visible (VIS), near-infrared (NIR), shortand mid-wave infrared (SMIR), and long-wave infrared (LWIR). For calibration, MODIS includes a set of onboard calibrators (OBC) such as a solar diffuser (SD) and a solar diffuser stability monitor (SDSM) for RSB calibration, ${ }^{3,4}$ a blackbody (BB) for TEB calibration ${ }^{5-7}$ and a spectroradiometric calibration assembly (SRCA) for sensor spectral and spatial characterization. ${ }^{8,9}$ The TEB are calibrated on-orbit on a scan-by-scan basis using a quadratic calibration algorithm by observing a large aperture V-grooved blackbody, which is controlled at a constant temperature of $290 \mathrm{~K}$ for Terra and $285 \mathrm{~K}$ for Aqua. A space view (SV) port is also used in order to determine the background signal for the MODIS bands.

Sentinel-3A, one major piece of the European Copernicus Space program, was launched on 16 February 2016 at an altitude of $815 \mathrm{~km}$ and a 27-day repeat cycle for observations with a 10:00 am local equatorial crossing time. The main objective of the Sentinel-3 mission is to accurately measure the sea surface topography, sea and land surface temperature, and ocean and land surface color. ${ }^{10}$ The Sentinel-3 mission is jointly operated

Further author information: (Send correspondence to A.S)

A.S: E-mail: ashish.shrestha@ssaihq.com, Telephone: 13018672071 
by European Space Agency (ESA) and European Organisation for the Exploitation of Meteorological Satellites (EUMETSAT) to support ocean forecasting systems, environmental monitoring and climate monitoring. The spacecraft carries four main instruments: a) Ocean and Land Color Instrument (OLCI), b) Sea and Land Surface Temperature Radiometer (SLSTR), c) Dual-frequency (Ku and C band) Synthetic Aperture Radar Altimeter (SRAL) and d) Microwave Radiometer.

SLSTR, the focus of this paper, is a multichannel scanning radiometer that is a follow-on to the two decades of successful operation of along-track scanning radiometer (ATSR) series. ${ }^{11}$ It was designed by incorporating the basic functionality of ATSR, with the addition of some new, more advanced, features. ${ }^{10}$ SLSTR has 11 spectral bands with wavelengths from 0.55 to $12 \mu \mathrm{m}$. The spatial resolution for VIS and SWIR bands $(S 1-S 6)$ is $500 \mathrm{~m}$, while IR and fire bands $(S 7-S 9$ and $F 1-F 2)$ have a $1 \mathrm{~km}$ resolution. SLSTR includes a set of on-board calibrators such as solar diffuser for calibrating the visible to short-wave infrared channels and two highly stable BB targets for calibrating the infrared channels. Among the two BB targets, one is maintained at a temperature lower than the expected surface temperature $(265 \mathrm{~K})$ while the other target is maintained at a temperature higher than the expected range $(302 \mathrm{~K})$, thus covering the full range of sea surface brightness temperatures. As a result, SLSTR can be regarded as a near-ideal radiometer. It is designed to have an uncertainty of $0.3 \mathrm{~K}$ for a $5^{\circ}$ by $5^{\circ}$ latitude-longitude area and a temporal stability of $0.1 \mathrm{~K} /$ decade. $^{10}$

Both MODIS instruments include various on-board calibrators. Although on-orbit results from BB demonstrate excellent short-term and long-term stability for both the Terra and Aqua MODIS, ${ }^{12}$ it is important to validate it with an independent instrument. Vicarious calibration methodologies are used as an alternative to the on-board calibration techniques for monitoring the sensor's performance. In this paper, the calibration consistency between MODIS bands 31 and 32 and SLSTR bands S8 and S9 is evaluated using vicarious calibration methods. In this approach, frequent and repeated observations of Dome Concordia (C), Antarctica $\left(75.12^{\circ} \mathrm{S}\right.$, $123.395^{\circ} \mathrm{E}$ ) are used to retrive the at-sensor radiance and brightness temperature (BT) ${ }^{13-15}$ The relative spectral response (RSR) for the matching MODIS and SLSTR bands is shown in Figure 1. Differences caused by mismatch in the spectral response function are not considered in this study. With the instrument background and objective defined, we briefly describe the target site (Dome C), data selection procedure and methodology used in the following sections. Results are discussed in Section 3.

\section{Spectral Response: Sentinel and MODIS}

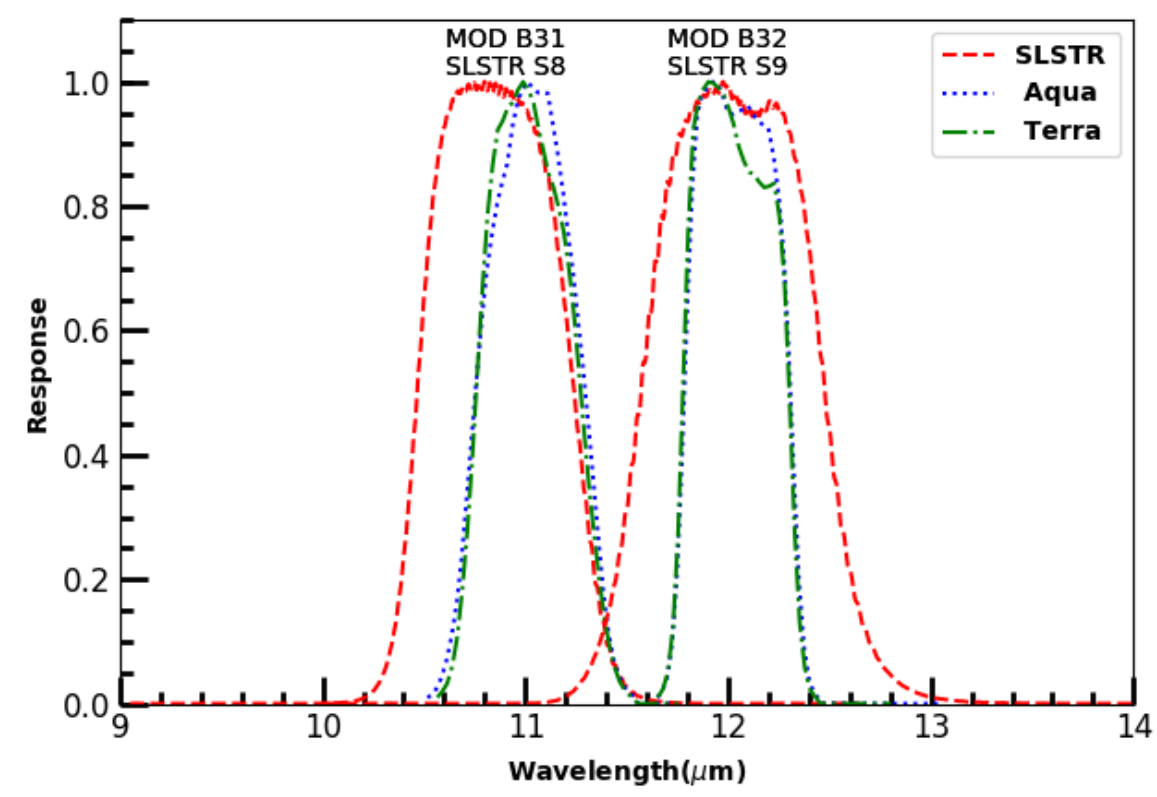

Figure 1: Relative spectral responses profiles for matching bands of MODIS and SLSTR. 


\section{SCENE SELECTION AND METHODOLOGY}

The Committee on Earth Observation Satellites-Working Group on Calibration and Validation (CEOS-WGCV) has identified a number of ground targets for validating the calibration consistency of satellite sensors. One such target used to track the on-orbit calibration of thermal remote sensing satellite instruments is Dome C. It is one of the most homogeneous Earth View (EV) targets and is situated near the Italian-French base of Concordia in a high polar plateau region with a mean elevation of $3200 \mathrm{~m}$. This site is well-suited for tracking the calibration stability and consistency of satellite sensors due to a number of factors such as relative uniformity, long-term stability, dry atmosphere, low aerosol loading and wind speed, low cloud cover and frequent satellite overpasses. ${ }^{16}$ Since 1995, the University of Wisconsin-Madison's Antarctic Meteorological Research Center (AMRC) has operated an Automatic Weather Station (AWS) at Dome C collecting various meteorological measurements at 10-minute intervals. ${ }^{17}$ The data is available through the University of Wisconsin's site (ftp://amrc.ssec.wisc.edu/pub/aws/). For all MODIS granules, the closest AWS measurements are retrieved. An example image from Terra MODIS band 31 is projected on an orthographic map of Antarctica with Dome $\mathrm{C}$ and is shown in Figure 2.

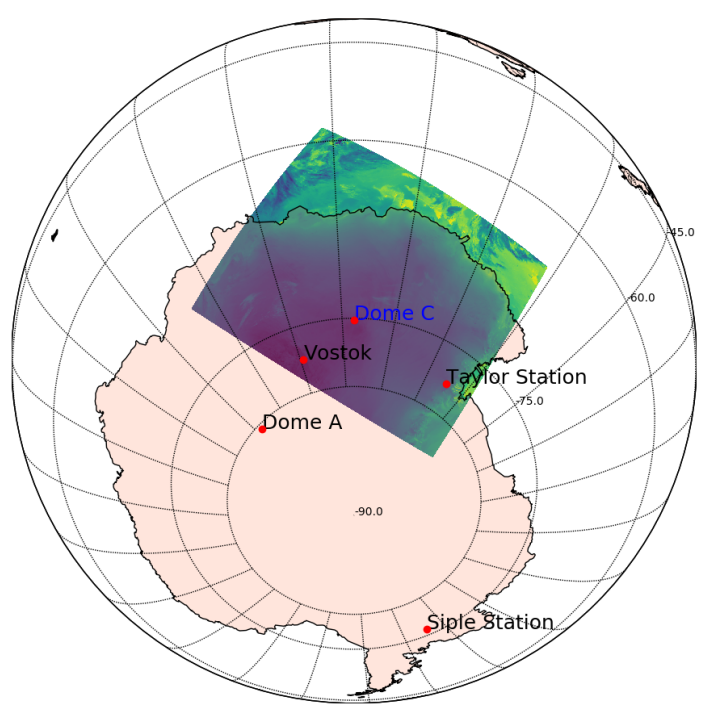

Figure 2: Map of Antarctic plateau with Terra MODIS band 31 image projected.

Both MODIS and Sentinel-3 are near sun-synchronous polar-orbiting sensors. Since Dome C is located near the Earth's south pole, all the instruments make several overpasses (typically $8-10$ ) over Dome C each day at different viewing angles. Typically the minimum and maximum overpass time difference between Aqua and Sentinel is about 1 and 183 minutes, respectively while for Terra and Sentinel it is 1 and 34 minutes. For MODIS, the radiance of a $20 \times 20 \mathrm{~km}$ region of interest (ROI) centered at the latitude and longitude of Dome $\mathrm{C}$ is retrieved from the level 1B (L1B) product (Collection 6.1). Using the Plank equation, the retrieved radiance is then converted to BT. The corresponding cloud mask product (MOD35_L2 for Terra, MYD35_L2 for Aqua) is used to identify and screen the cloudy pixels. As both MODIS and SLSTR have same spatial resolution $(1 \mathrm{~km})$, for SLSTR, BT for the same ROI $(20 \times 20)$ was retrieved using the L1B product. To assess the calibration consistency between MODIS and SLSTR, the AWS data closest in time to the corresponding instrument observation is separately recorded (each AWS sample is an average of 10 minutes of data). The temperature difference between each instrument band and AWS is calculated. Finally, using a double difference between SLSTR and MODIS, the relative bias is calculated. No atmospheric correction is considered for this study, but the impacts to these window channels is expected to be minimal. For some of our analysis, to cover the entire range of BT, scenes from other ground targets are also selected. More description about the additional ground targets is provided in the next section. 


\section{RESULTS}

Considering all viewing geometry, the total number of acquisitions is 2989 for Terra, 2963 for Aqua and 1961 for SLSTR. In Figure 3, we show band-averaged BT trend for matching bands, i.e. bands 31 and 32 of MODIS and bands S8 and S9 of SLSTR, respectively. The blue circles, green triangles and red diamonds represent the BT for Aqua, Terra and SLSTR, respectively. Standard deviation for each point is also shown on the right side of the figure. Based on the plot, it is evident that the BT from each instrument follows the same pattern, i.e. a temperature peak in January and a minima in July. For SLSTR band S8, the BT is higher during polar winter (May-August) compared to the BT of MODIS band 31. No obvious differences are apparent for MODIS band 32 and SLSTR band S9.
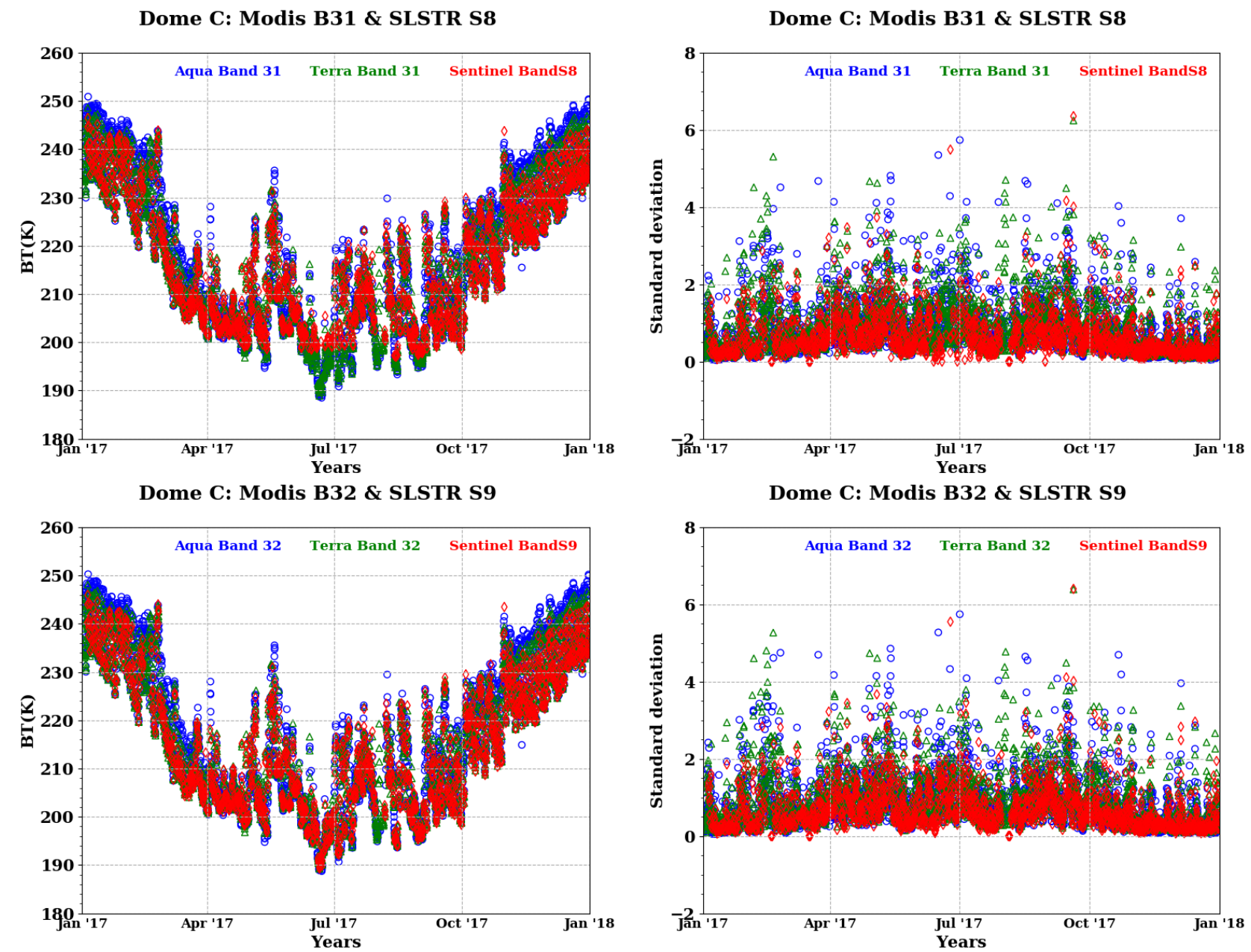

Figure 3: BT trend and standard deviation for Aqua (blue cricle), Terra (green triangle) and SLSTR (red diamond) for matching bands.

When considering only the near-simultaneous scenes, or scenes with time differences of less than 30 minutes between two sensors, the total number of acquisitions for Terra and SLSTR is 495, while the Aqua and SLSTR has 181 pairs. In Figure 4, we show the comparison of BT measurements obtained from matching bands, i.e. bands 31 and 32 of MODIS and bands S8 and S9 of SLSTR, respectively. These plots relate BT obtained from MODIS bands to the corresponding BT obtained from SLSTR bands. The MODIS BT is plotted on the $x$ axis, and the SLSTR BT is plotted on the $y$ axis. A linear regression line was fitted to the data and a T-test was performed at the confidence interval of $95 \%$. Fit statistics for matching bands of MODIS and SLSTR are shown 
in Table 1. The black line represents the one-to-one relationship that indicates perfect agreement between the BT measurements. Figure 4 shows that for MODIS band 31 and SLSTR band S8, the BTs less than $200 \mathrm{~K}$ start to deviate from the one-to-one line, indicating some discrepancies at the lower end of the curve. Although the exact reason for the discrepancies between S8 and band 31 is not understood, we find that during the polar winter (May-August), number of valid pixel for sentinel S8 are considerably smaller compared to S9.
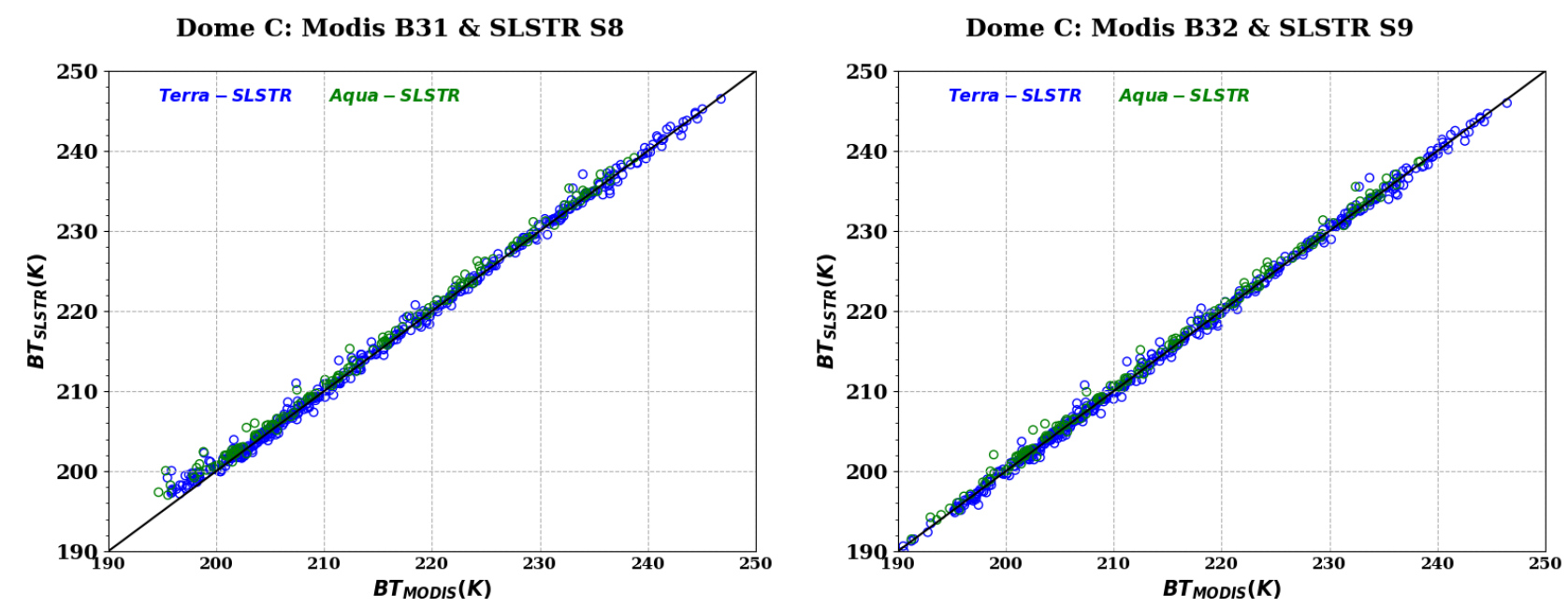

Figure 4: Comparison of BT for matching bands of MODIS and SLSTR.

\begin{tabular}{|c|c|c|c|c|}
\hline \multirow{2}{*}{} & \multicolumn{2}{|c|}{ S8/B31 } & \multicolumn{2}{c|}{ S9/B32 } \\
\cline { 2 - 5 } & Aqua & Terra & Aqua & Terra \\
\hline Slope/P-value & $0.9799 / 0.0000$ & $0.9798 / 0.0000$ & $0.9972 / 0.0000$ & $1.0090 / 0.0000$ \\
\hline Bias/P-value & $5.0690 / 0.0000$ & $4.9720 / 0.0000$ & $1.0890 / 0.2300$ & $-0.1800 / 0.6800$ \\
\hline $\mathbf{R}^{\mathbf{2}}$ & 0.996 & 0.997 & 0.997 & 0.998 \\
\hline
\end{tabular}

Table 1: Fit statistics for the matching bands of MODIS and SLSTR.

In Figure 5, we show the band-averaged BT difference, $\Delta T$, between the two instruments. The MODIS BT is plotted on the $x$ axis and the BT differences between MODIS and SLSTR is plotted on the $y$ axis. Since the Dome $\mathrm{C}$ temperature varies between $185-250 \mathrm{~K}$, we added simultaneous scene pairs from other ground targets to cover the entire BT range. Of these pairs, 93 scenes for SLSTR and Terra were obtained from the Libya 4 site $\left(28.55^{\circ} \mathrm{N}, 23.39^{\circ} \mathrm{E}\right)$ while 78 pairs were obtained from a latitude range of $\pm 70^{\circ}$. No simultaneous scenes were found for Aqua and SLSTR from these additional sites. For each of the additional scenes pair, the BT is retrieved for a $20 \times 20 \mathrm{~km}$ ROI centered at the latitude and longitude of the corresponding site. Finally, $\Delta T$ for each matching band was calculated by

$$
\Delta T=B T\left(b_{M}\right)-B T\left(b_{S L S T R}^{\prime}\right)
$$

where, $b_{M}$ is the Terra or Aqua MODIS band, $b_{S L S T R}^{\prime}$ is the corresponding SLSTR band. The circles, triangles and diamonds represent the BT difference calculated from the scene pair at Dome C, latitude $\pm 70^{\circ}$ and Libya 4 , respectively. Ideally, the BT differences should be close to zero over the entire range of BT (assuming a small impact due to the difference in spectral responses of MODIS and SLSTR). However, for MODIS band 31 and SLSTR band S8, a larger difference was seen at the lower end of the temperature range (less than $200 \mathrm{~K}$ ), indicating some temperature dependency. No obvious differences were apparent for MODIS band 32 and SLSTR band S9. Mean values calculated from the dataset are listed in Table 2. Since BT difference for additional 
ground targets are little higher compared to BT differences from Dome C, mean for all the dataset as well as mean without considering the additional ground targets are both listed in Table 2 .
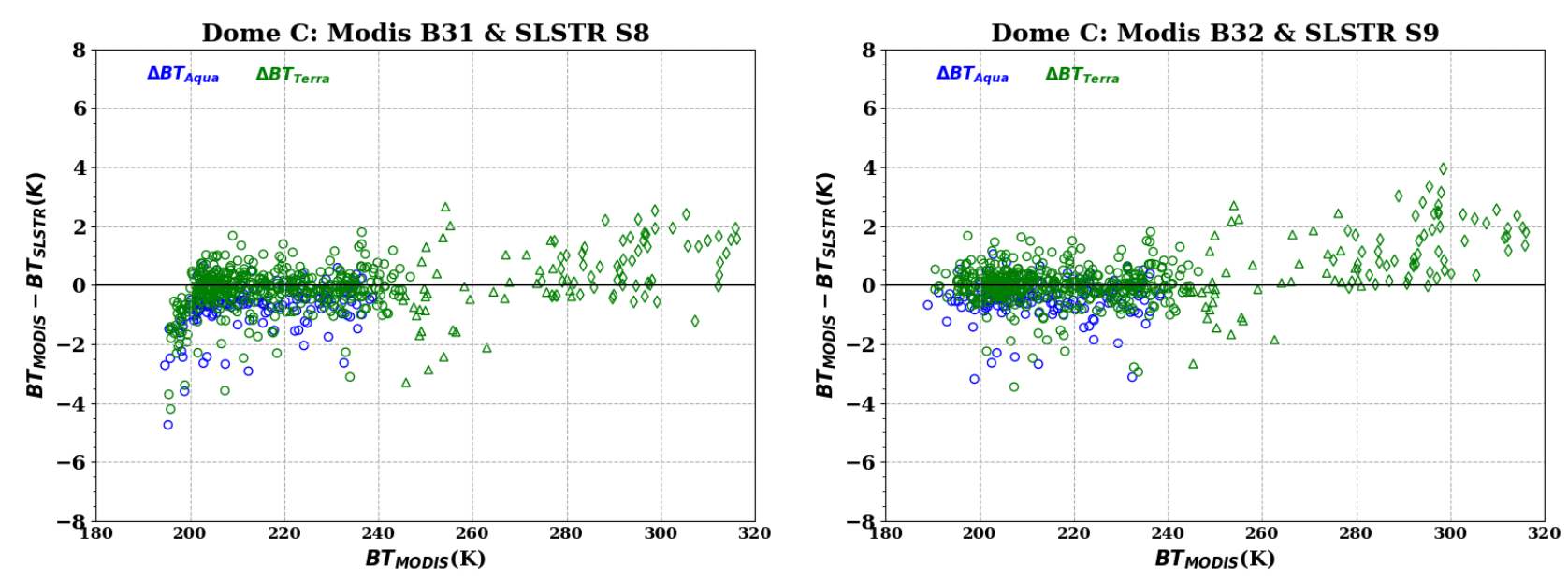

Figure 5: BT difference trend for the matching bands of MODIS and SLSTR.

\begin{tabular}{|c|c|c|c|c|}
\hline \multirow{2}{*}{} & \multicolumn{2}{|c|}{ S8/B31 } & \multicolumn{2}{c|}{ S9/B32 } \\
\cline { 2 - 5 } & Aqua & Terra & Aqua & Terra \\
\hline Mean (with all the ground target) & $-0.84 \mathrm{~K}$ & $0.018 \mathrm{~K}$ & $-0.167 \mathrm{~K}$ & $0.276 \mathrm{~K}$ \\
\hline Mean(without additional ground targets) & $-0.84 \mathrm{~K}$ & $-0.195 \mathrm{~K}$ & $-0.167 \mathrm{~K}$ & $-0.005 \mathrm{~K}$ \\
\hline
\end{tabular}

Table 2: Mean Values for the matching bands of MODIS and SLSTR.

Figure 6 shows the same data used in Figure 5 plotted by binning the BT difference between MODIS and SLSTR at every $10 \mathrm{~K}$. The error bar for each bin is also provided. This clearly indicates that at cold scene temperatures, MODIS band 31 and SLSTR band S8 have a bias of upto $2 \mathrm{~K}$.
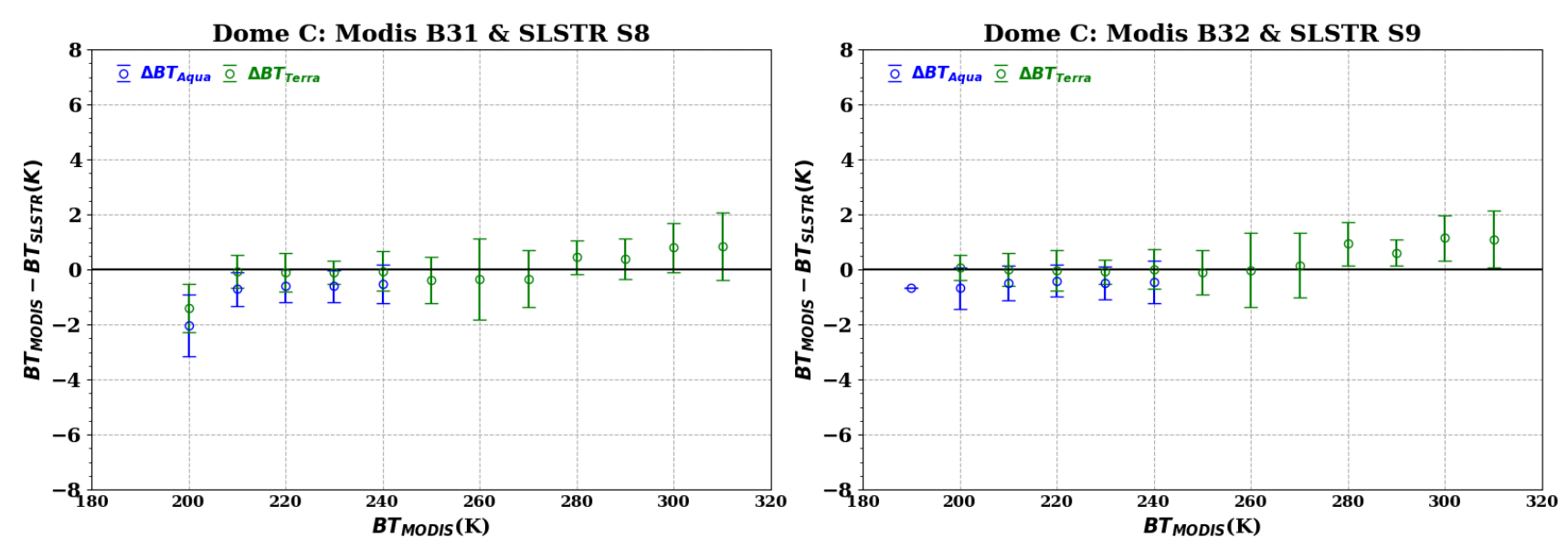

Figure 6: BT difference (with binning) trend using the same data as Figure 5. 
To assess the calibration consistency between MODIS and SLSTR, a double difference, or relative bias between MODIS and SLSTR, is assessed using all Dome C scenes. The relative bias is calculated by

$$
\text { Relative Bias }=\Delta T_{\text {Modis }}-\Delta T_{\mathrm{SLSTR}}
$$

$\Delta T$ for each instrument is calculated by

$$
\Delta T_{\text {Instr }}=B T_{\text {Instr }}-\text { AWS }
$$

Since the MODIS and SLSTR scenes used for this analysis are not coincident, to minimize the impact of overpass time differences, the BT differences are averaged over each week. In Figure 7, we show the relative bias trend for matching bands of MODIS and SLSTR. It is evident that a temperature dependency exists at the lower end of the BT range for MODIS band 31 and SLSTR band S8. Figure 7 also shows that the relative bias remains stable and approximately linear across the entire BT range for MODIS band 32 and SLSTR band S9.
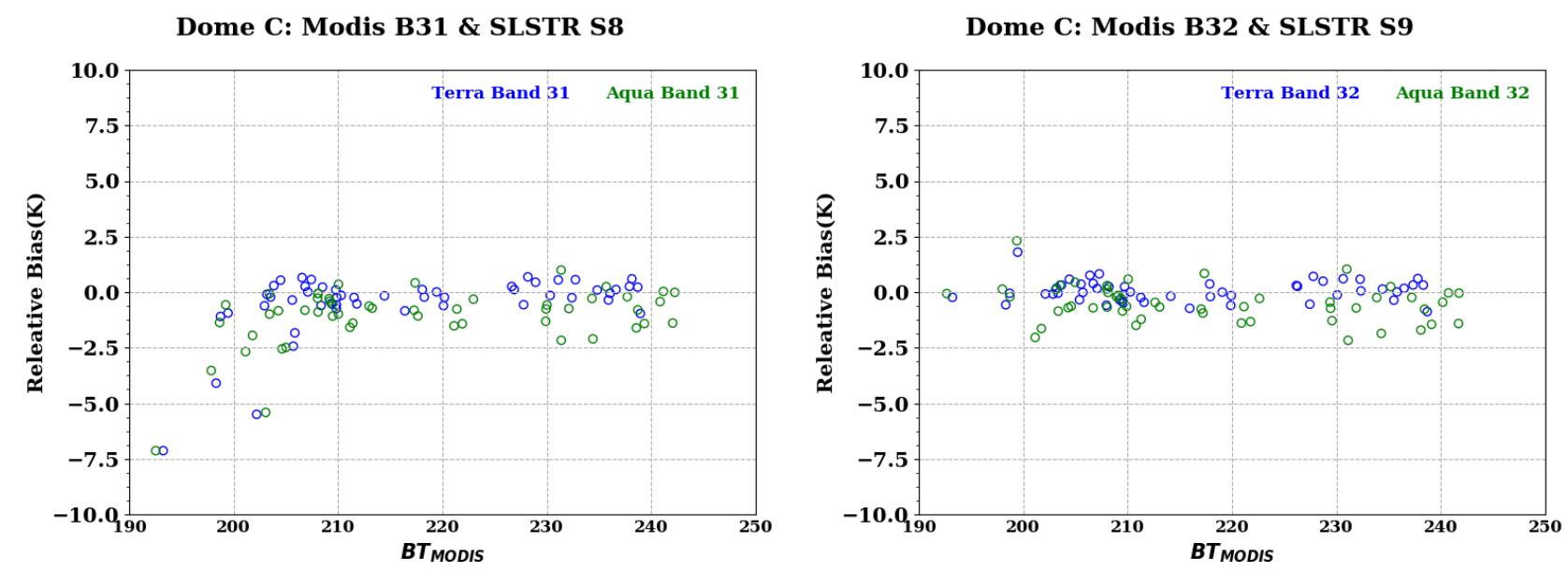

Figure 7: Relative bias trend for matching bands of MODIS and SLSTR.

\section{SUMMARY}

Both MODIS instruments are currently operating beyond their design lifetimes and continue to provide reliable scientific measurements. Vicarious calibration methodologies are used as an alternative to the on-board calibration techniques for monitoring the sensor's performance. SLSTR is a follow-on of the ATSR sensor with the addition of some new, more advanced features. In this paper, we use techniques previously developed, to evaluate the calibration stability and consistency of MODIS band 31 and 32 and SLSTR band S8 and S9. The results indicate that for MODIS band 31 and SLSTR band S8, a difference of up to $2 \mathrm{~K}$ exists at the lower BT range (less than $200 \mathrm{~K}$ ). No obvious difference was observed for MODIS band 32 and SLSTR band S9. While the result presented only focused on MODIS bands 31 and 32 and SLSTR bands S8 and S9, we plan to extend this analysis to evaluate calibration consistency of matching RSB of MODIS and SLSTR in the future.

\section{ACKNOWLEDGMENTS}

The authors would like to thank members of the MODIS Characterization Support Team in particular Emily Aldoretta for her comments and review of this work.

Copyright contains modified Copernicus Sentinel data (2017-18), processed by ESA. 


\section{REFERENCES}

[1] Barnes, W. L. and Salomonson, V. V., "Modis: A global image spectroradiometer for the earth observing system," Crit. Rev. Opt. Sci. Technol. CR47, 285-307 (1993).

[2] Salomonson, V., Barnes, W., Xiong, X., Kempler, S., and Masuoka, E., "An overview of earth observing system modis instrument and associated data systems performance," Proc. IGARSS 2, 1174-1176 (2002).

[3] Xiong, X., Sun, J., Barnes, W., Salomonson, V., Esposito, J., Erives, H., and Guenther, B., "Multiyear on-orbit calibration and performance of terra modis reflective solar bands," IEEE Trans. Geosci. Remote Sens. 45(4), 879 - 889 (02 April 2007).

[4] Xiong, X., Sun, J., Xie, X., Barnes, W., and Salomonson, V., "On-orbit calibration and performance of aqua modis reflective solar bands," IEEE Trans. Geosci. Remote Sens. 48(1), 535 (2009).

[5] Xiong, X., Chiang, K., Wu, A., Barnes, W., Guenther, B., and Salomonson, V., "Multiyear on-orbit calibration and performance of terra modis thermal emissive bands," IEEE Trans. Geosci. Remote Sens. 46(6), 1790-1803 (16 May 2008).

[6] Xiong, X., Wenny, B., Wu, A., Barnes, W., and Salomonson, V., "Aqua modis thermal emissive band onorbit calibration, characterization, and performance," IEEE Trans. Geosci. Remote Sens. 47(3), 803-814 (12 December 2008).

[7] Xiong, X., Wu, A., Wenny, B., Madhavan, S., Wang, Z., Li, Y., Chen, N., Barnes, W., and Salomonson, V., "Terra and aqua modis thermal emissive bands on-orbit calibration and performance," IEEE Trans. Geosci. Remote Sens. 53(10), 5709 (2015).

[8] Xiong, X., Che, N., and Barnes, W., "Terra modis on-orbit spatial characterization and performance," IEEE Trans. Geosci. Remote Sens. 43(2), 355-365 (24 January 2005).

[9] Xiong, X., Che, N., and Barnes, W., "Terra modis on-orbit spectral characterization and performance," IEEE Trans. Geosci. Remote Sens. 44(8), 2198-2206 (24 July 2006).

[10] Sentinel-3 Team, [Sentinel-3 User Handbook], European Space Agency (2013).

[11] Smith, D. L., Nightingale, T. J., Mortimer, H., Middleton, K., Edeson, R., Cox, C., Mutlow, C., Maddison, B., and Coppo, P., "Calibration approach and plan for the sea and land surface temperature radiometer," Journal of Applied Remote Sensing 8(1), 084980 (2014).

[12] Xiong, X., Wenny, B., Wu, A., and Barnes, W., "Modis onboard blackbody function and performance," IEEE Trans. Geosci. Remote Sens. 47(12), 4210 (2009).

[13] Wenny, B. and Xiong, X., "Using a cold earth surface target to characterize long-term stability of the modis thermal emissive bands," IEEE Geosci. Remote Sens. Lett 5, 162165 (2008).

[14] Wenny, B., Xiong, X., and Madhavan, S., "Evaluation of terra and aqua modis thermal emissive band calibration consistency," Proc. SPIE, 8533 (2012).

[15] Wenny, B., Xiong, X., Madhavan, S., Wu, A., and Li, Y., "Long-term band-to-band calibration stability of modis thermal emissive bands," Proc. SPIE, 8724 (2013).

[16] Six, D., Fily, M., Alvain, S., Henry, P., and Benoist, J., "Surface characterisation of the dome concordia area (antarctica) as a potential satellite calibration site, using spot 4/vegetation instrument," Rem. Sens. Environ. 89, 83-94 (2004).

[17] Stearns, C. R., Keller, L., Weidner, G., and Sievers, M., "Monthly mean climatic data for antarctic automatic weather stations," Antarctic Research Series 61, 1-22 (1993). 